\title{
Effect of protein isotope labeling on the catalytic mechanism of lactate
}

\section{dehydrogenase}

Tsuyoshi Egawa,,+ Hua Deng,,+ Eric Chang, \$ and Robert Callender $\$$

\$Department of Biochemistry, Albert Einstein College of Medicine, Bronx, NY 10461 and

\$Department of Chemistry and Physical Sciences, Pace University, New York, NY 10038

\section{Supplement}

In isotope edited difference FTIR studies, two spectra will be obtained from two enzyme complexes prepared with unlabeled and ${ }^{13} \mathrm{C} 2$ labeled substrate. Their difference spectrum should only retain the IR bands that are affected by the ${ }^{13} \mathrm{C}$ label, while all other bands, including protein bands, should be eliminated. However, artifacts may arise from imperfect balance between the two samples, such as mismatch in sample concentration, in different hydration levels in the two deuterated samples, etc, resulting in uneven backgrounds and/or features which are due to protein bands but may look like substrate peaks in the difference spectrum. ${ }^{1}$ One way to identify the false pyruvate bands is to use different subtraction factors as shown in Figure S1. The real pyruvate bands do not disappear with small variation of the subtraction factor while the bands from protein are much more sensitive to such change. 

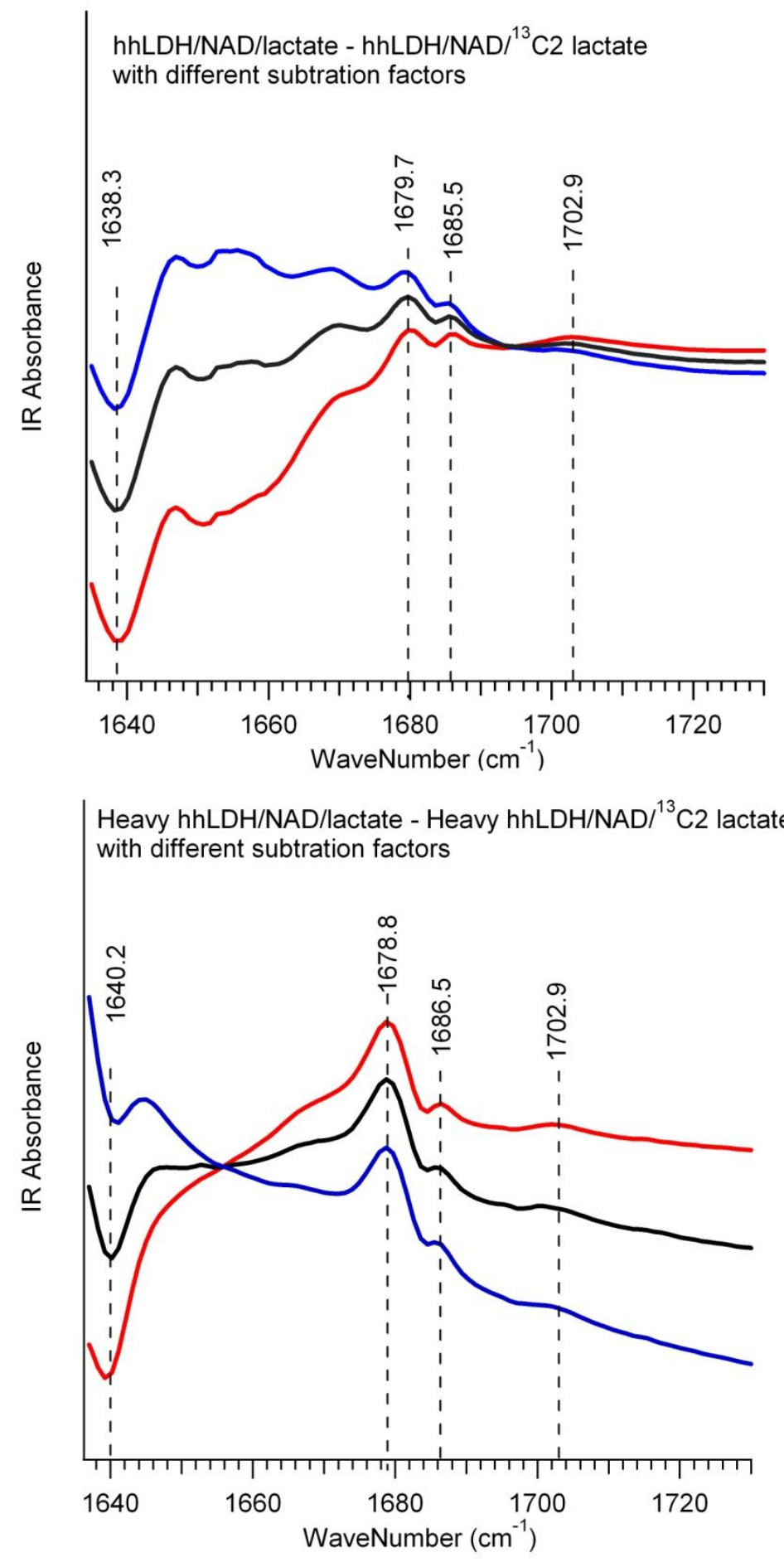

Figure S1. Difference IR spectra of pyruvate minus ${ }^{13} \mathrm{C} 2$ pyruvate with three different subtraction factors in light hhLDH•NADH•pyruvate (top spectra) and in Heavy hhLDH•NADH•pyruvate (bottom spectra). Starting concentration of reactants enzyme, NAD, lactate were 7:7:80 $\mathrm{mM}$. The samples were prepared in $100 \mathrm{mM}$ phosphate buffer at $\mathrm{pH} 7$ (uncorrected $\mathrm{pH}$ meter reading) in $\mathrm{D}_{2} \mathrm{O}$ at $20{ }^{\circ} \mathrm{C}$. Three subtraction factors $0.97,1.00$ and 1.03 were used to generate the spectra shown in the Figure. 
Pyruvate band assignments in the difference FTIR spectra.

In the light hhLDH difference spectra (Figure S1, top), the broad band between $1650-1660 \mathrm{~cm}^{-1}$ disappears when larger subtraction factor is used, indicating that it is a protein band. The bands with frequency labels are not affected by small changes of the subtraction factors, thus can be assigned to pyruvate bands. In the heavy hhLDH difference spectra (Figure S1, bottom), the IR bands with frequency labels are also not affected by the changes of subtraction factor and thus can be assigned to pyruvate bands. These pyruvate frequencies, including the small differences in the light and heavy enzyme complexes are very reproducible and also reported in our previous studies on the pig heart LDH. ${ }^{2}$ The resolution in this area is better than $0.5 \mathrm{~cm}^{-1} .^{2}$

1. Deng, H.; Vu, D. V.; Clinch, K.; Desamero, R.; Dyer, R. B.; Callender, R., Conformational heterogeneity within the Michaelis complex of lactate dehydrogenase. J. Phys. Chem. B 2011, 115, 7670-6778.PMC3111758

2. Peng, H.-L.; Deng, H.; Dyer, R. B.; Callender, R., The Energy Landscape of the Michaelis Complex of Lactate Dehydrogenase: Relationship to Catalytic Mechanism. Biochemistry 2014, 53, 1849-1857.PMC3985751 